\title{
Teleophthalmology and the digital divide: inequities highlighted by the COVID-19 pandemic
}

\author{
Angelica C. Scanzera $\mathbb{1}^{1} \cdot$ Sage J. Kim² $\cdot$ R. V. Paul Chan ${ }^{1}$
}

Received: 7 October 2020 / Revised: 19 October 2020 / Accepted: 12 November 2020 / Published online: 30 November 2020

(c) The Royal College of Ophthalmologists 2020

\begin{abstract}
The COVID-19 pandemic has brought an unprecedented change in the way we deliver eye care to our patients, most notably with the rapid addition of telehealth technology into our practices. We have welcomed telehealth with open arms in hopes that it would improve access to care for our patients; however, one question remains: Does it improve health equity?
\end{abstract}

\section{Adapting to telehealth}

Initial factors driving telehealth adaptation in eye care during the pandemic included (1) shelter-in-place orders, (2) changes in reimbursement for telehealth services, and (3) recommendations by state and national organizations to cancel non-urgent visits and procedures. Telehealth in eye care is not a novel topic, as store-and-forward systems have proved successful in the management of diabetic retinopathy and Retinopathy of Prematurity [1]. However, virtual video consultations are just recently becoming more prevalent.

Ophthalmology departments have remained flexible while adapting to telehealth. The Department of Ophthalmology and Visual Sciences at the University of Illinois at Chicago (UIC) primarily utilized store and forward technology for diabetic retinopathy screenings prior to COVID19. In response to the pandemic, a tele-triage system was initiated to appropriately triage urgent patients into our clinic [2]. Video visits continue to be offered to patients, with some specialties, such as neuro-ophthalmology and oculoplastics, adapting to the technology faster than others. To increase the quality of a virtual visit, hybrid models are

Angelica C. Scanzera

ascanz@uic.edu

1 Department of Ophthalmology and Visual Sciences, Illinois Eye and Ear Infirmary, University of Illinois at Chicago, $1855 \mathrm{~W}$. Taylor Street, Chicago, IL 60612, USA

2 Division of Health Policy \& Administration, School of Public Health, University of Illinois at Chicago, 1603 W. Taylor Street, Chicago, IL 60612, USA being introduced, allowing critical exam components such as intraocular pressure, visual fields, or imaging to be obtained in-person prior to a video visit. This technology has allowed more patients to be evaluated, but access to all patients is unproven.

In a survey approved by the Institutional Review Board at UIC, patients determined by a provider to require an urgent visit through our tele-triage system were asked whether they would be interested in a telehealth visit if deemed appropriate by their provider. Of 216 patients who responded, $104(48.1 \%)$ had interest in a telehealth visit. Patients interested were more likely to be younger (42.6 vs. 50.3 years; $p=0.002$ ), and women (OR 1.82 [CI 1.062, 3.164]; $p=0.0295)$; However, no difference was noted by race $(p=0.712)$ or insurance status $(p=0.252)$.

\section{Health equity and the digital divide}

Our health system serves residents of communities who primarily identify as racial and ethnic minorities (29\% Black and 35\% Latinx), $21 \%$ of patient households' income is less than 20,000, and $46 \%$ is less than 40,000 . These communities have greater rates of unemployment and uninsured persons than the average in the State of Illinois and the United States [3]. UI Health identifies three priority areas including addressing social and structural determinants of health, improving access to care and community resources, and prevention of chronic diseases, which are designed to focus on improving health equity in UI Health's catchment areas. Unfortunately, the same communities directly affected by COVID-19 are also those given unequal opportunities to benefit from telehealth. One report on the 
use of telehealth in chronic disease management during COVID-19 found that while Whites had an increased proportion of patient visits after the introduction of telemedicine, Blacks and Latinx had a decrease in the proportion of visits [4]. Access to a virtual at-home visit requires a computer or smartphone, internet access, and the knowledge of how to use it. These are resources often taken for granted, though the likelihood of owning a smartphone and having home broadband internet available is lower in Black and Latinx adults, individuals over 65 years old, and those with low-income levels [5]. In addition to the aging population, Blacks and Latinx are also two and three times more likely to lack digital literacy compared to their White counterparts [6]. Visual impairment also limits the ability to perform an eye exam via telehealth, as patients may have difficulty reading font on the screen or opening certain applications. Visual impairment increases with age, and Blacks and Latinx are more likely to be visually impaired than Whites in the United States [7]. This continues to further the divide, and solely offering telehealth does not guarantee access to the technology. For example, many of our providers have reported connectivity or hardware issues that caused the visit to be conducted over the phone, limiting the quality of the visit.

\section{Next steps}

It is widely recognized that an individual's health is determined primarily by what happens outside of the healthcare system, such as poverty and lack of access to care [8]. We must continue to recognize these broader social inequities, as they contribute to poorer health outcomes. Possible programs relevant to telehealth include community-led internet service subsidy programs or WIFI hot-spot lending programs, programs to refurbish and redistribute smartphone devices, and individual training programs in the use of digital health tools. Virtual video visits can only be effectively utilized when access to technology and the internet is equally improved.

There is no doubt that telehealth is vital in providing care to patients in all specialties during COVID-19, and there are also changes we can make in healthcare delivery to promote health equity in eye care. For starters, providing coordinators to educate on the use of digital health tools can be directly implemented into our practices. In addition, doctorto-doctor consults between emergency departments, primary care physicians, optometrists, and ophthalmologists can increase access to care by reducing the number of visits a patient makes for the management of eye disease. Investing in technology in these settings, such as fundus cameras or remote slit lamps [9] could help us maintain parity of care. These improvements have the potential to both limit unnecessary exposure to COVID-19 and improve access to care in communities lacking resources and subspecialty care far beyond the current pandemic.

With telehealth expanding in ophthalmology, its full potential has yet to be realized. Telehealth is required to respond to COVID-19, but this technology will also play a crucial role in the future of eye care. We have a unique opportunity to expand telehealth to improve access to eye care. Investing in the proper strategic partnerships and technology could advance health equity, and eye care providers have a responsibility to continue adapting to these changes for the good of our communities.

\section{Disclaimer}

The views expressed in this manuscript are those of the authors and do not necessarily represent the views of the National Eye Institute; the National Institute on Minority Health and Health Disparities; the National Institutes of Health; or the U.S. Department of Health and Human Services.

Acknowledgements The authors thank the Center for Health Equity Research (CHER) Chicago for their invaluable collaboration.

Funding This work was supported by funding by NIH/NEI K12 EY021475 (Scanzera), NIH/NEI EY001792, NIH/NEI R01 EY029673 (Chan), NIH/NIMHD U54MD012523 (Kim, Scanzera), and an unrestricted grant to the Department of Ophthalmology and Visual Sciences from Research to Prevent Blindness. Dr. R.V. Paul Chan discloses the following (1) Alcon; (2) Novartis; (3) Phoenix (Unpaid $\mathrm{SAB})$. The following authors have no financial disclosures: Dr. Angelica C. Scanzera and Dr. Sage Kim. All authors attest that they meet the current ICMJE criteria for authorship.

\section{Compliance with ethical standards}

Conflict of interest The authors declare that they have no conflict of interest.

Publisher's note Springer Nature remains neutral with regard to jurisdictional claims in published maps and institutional affiliations.

\section{References}

1. Chee RI, Darwish D, Fernandez-Vega A, et al. Retinal telemedicine. Curr Ophthalmol Rep. 2018;6:36-45.

2. Scanzera AC, Cole E, Valikodath NG, et al. Implementation of COVID-19 Protocols and Tele-Triage in an Academic Ophthalmology Department. J Acad Ophthalmol. 2020;12:e151-8.

3. UI Health. 2019 Community Assessment of Needs: Towards Health Equity. University of Illinois Hospital and Health Sciences System: Chicago: University of Illinois; 2019. 
4. Nouri S, Khoong EC, Lyles CR, Karliner L. Addressing Equity in Telemedicine for Chronic Disease Management During the COVID-19 Pandemic. N Engl J Med: Catalyst Innovations in Care Delivery. 2020. https://catalyst.nejm.org/doi/full/10.1056/CAT.20. 0123.

5. Pew Research Center. Internet/Broadband Fact Sheet. August, 20202019.

6. Mamedova S, Pawlowski E. Stats in Brief: A Description of U.S. Adults Who Are Not Digitally Literate. National Center for Education Statistics. 2018.
7. Zambelli-Weiner A, Crews JE, Friedman DS. Disparities in adult vision health in the United States. Am J Ophthalmol. 2012;154: S23-30.e21.

8. County Health Rankings Model. County Health Rankings \& Roadmaps. 2019. https://www.countyhealthrankings.org/explorehealth-rankings/measures-data-sources/county-health-rankingsmodel.

9. Nankivil D, Gonzalez A, Rowaan C, Lee W, Aguilar MC, Parel JA. Robotic remote controlled stereo slit lamp. Transl Vis Sci Technol. 2018;7:1. 